\title{
Real-time Pricing and Distributed Decision Makings Leading to Optimal Power Flow of Power Grids
}

\author{
Kenji Hirata, João P. Hespanha, and Kenko Uchida
}

\begin{abstract}
Motivated by distributed control problems of power supply/demand networks, this paper investigates application case studies of the real-time pricing and distributed decision makings methodology. We consider the optimal power flow problem with the DC power flow model, and the New England 39-bus test system is used. Stability of the resulting price based control system is analyzed with consideration for specific structures of the power flow problem. The resulting simulation studies illustrate the efficiency of the proposed method and validate the stability analysis procedure.
\end{abstract}

\section{INTRODUCTION}

Control design problems usually consider regulation and tracking of system states with respect to known set-points or trajectories. Optimal set-points or trajectories are typically given or determined a priori by solving an appropriate optimization problem. The optimization problem may incorporate economical efficiency, security limits of the plant and operating constraints associated with steady-state operation of the entire system. The control system may be required to follow a time-varying demand, and the optimization problem should be solved again to reflect new demand conditions and update optimal set-points or trajectories in real-time.

If one considers a large scale control system, electrical power supply/demand network for example, an appropriate optimization problem to determine optimal operating conditions becomes large, and solving the problem will be a nonimmediate task, especially in real-time. The components of the entire system may also be distributed, e.g., distributed generators and consumers over the power supply/demand network. Each component has own individual economical profit and wants to determine its optimal operating condition according to the optimization of individual profit, while the solution should satisfy specified constraints for social benefits, e.g., supply/demand balancing in steady-state over the power girds. Accordingly, a distributed mechanism somehow determines the operating condition that is a solution to the constrained optimization problem.

This material is based upon work supported by the Japan Science and Technology Agency, CREST, the Japan Society for the Promotion of Science under Grant No. 26420411, and the National Science Foundation under Grant No. CNS-1329650.

K. Hirata is with the Department of Mechanical Engineering, Nagaoka University of Technology, Nagaoka 940 2188, Japan.

J. P. Hespanha is with the Center for Control, Dynamical Systems, and Computation, and the Department of Electrical and Computer Engineering, University of California, Santa Barbara, CA 93106 USA.

$\mathrm{K}$. Uchida is with the Department of Electrical Engineering and Bioscience, Waseda University, Tokyo 169 8555, Japan.

K. Hirata and K. Uchida are with the Japan Science and Technology Agency, CREST.
The problem of selecting an economically efficient operating condition has been considered in [1], [2], [3], [4] and references therein. In [1], [2], the problem is investigated in non-distributed fashion. A dynamic controller based on the Karush-Kuhn-Tucker (KKT) optimality conditions has been proposed in [1], and a solution that uses penalty and barrier function to deal with constraints has been considered in [2]. The dynamic KKT controller has also been applied to the DC power flow control problem of power girds in [3], where it has also been shown that the dynamic KKT controller can be implemented in a distributed fashion to the DC power flow control problem. The problem has also been investigated in a distributed manner using passivity based techniques [4].

Motivated by price based approaches in power balancing [5], [6], [7], [8], [9], [10], [8], [11], [12], [13], [14], [3], [4], the authors have also proposed a real-time pricing strategy for selecting economically efficient operating conditions in [15]. In the proposed problem settings, each agent, a dynamic component of the entire large scale network of systems, is allowed to determine its own economically efficient optimal set-point according to its individual profit. On the other hand, the utility, which corresponds to an independent public commission, tries to realize a socially optimal solution that fulfills steady-state operating constraints. In order to align the individual decision making of each agent with the socially optimal solution, the utility is allowed to provide additional price, which conceptually represents tax to the agent or subsidy from the community, and each agent will participate to the community through optimization of the individual profit including the additional price. The resulting price based control system eventually realizes tracking to the socially optimal solution, in which no-one needs to solve a large scale optimization problem nor consider iterative calculations to determine the price.

This paper investigates an application case study of the real-time pricing and distributed decision makings methodology proposed in [15]. We consider the optimal power flow problem with the DC power flow model, and, to illustrate the potential of the developed methodology for practical application, we apply the proposed method to the widely used New England 39-bus test system [16] (see Fig. 2). Stability of the resulting closed-loop system, which consists of the real-time pricing strategy, distributed decision makings by each agent and generators/loads dynamics (see Fig. 1), are also analyzed with consideration for specific structures of the power flow problem. The simulation results illustrate the efficiency of the proposed method and validate the stability analysis procedure. 


\section{Problem Formulation}

\section{A. Dynamics of each Agent}

We consider a group of $n$ agents, $G_{i}, i \in N=\{1, \ldots, n\}$. Each agent $G_{i}$ has a group of other agents, who are called as the neighbors of $G_{i}$, and we denote by $N_{i} \subset N$ the set of indices of the neighbors of $G_{i}$. The agent $G_{i}$ has interactions between its neighbors and is represented by equation of the form

$$
\begin{aligned}
\dot{x}_{i}(t) & =f_{i}\left(x_{i}(t), z^{i}(t), w_{i}(t), r_{i}(t)\right) \\
z_{i}(t) & =g_{i}\left(x_{i}(t), z^{i}(t), w_{i}(t)\right)
\end{aligned}
$$

where $x_{i} \in X_{i} \subset \mathbb{R}^{n_{i}}$ denotes the state, $r_{i} \in R^{m_{i}}$ denotes the reference input, $z_{i} \in R^{m_{i}}$ denotes the output to be tracked to $r_{i}, w_{i} \in W_{i} \subset \mathbb{R}^{m_{w i}}$ denotes an exogenous input and $z^{i} \in \mathbb{R}^{m^{i}}$ represents interactions between the agents, where we set $x^{i} \in X^{i}=\prod_{j \in N_{i}} X_{i}$ and consider

$$
z^{i}(t)=g^{i}\left(x^{i}(t)\right)
$$

We suppose that each function in (1) is differentiable as is required.

\section{B. Distributed Determinations of Optimal Set-point}

We set an optimization problem for each $G_{i}, i \in N$ and suppose that the economically optimal set-point is given as its optimal solution.

$$
\min _{r_{i}} J_{i}\left(w_{i} ; r_{i}\right)
$$

subject to $h_{i j}\left(w_{i} ; r_{i}\right) \leq 0 \quad j \in T_{i}=\left\{1,2, \ldots, t_{i}\right\}$

where, for each given $w_{i} \in W_{i}, J_{i}\left(w_{i} ; \cdot\right): \mathbb{R}^{m_{i}} \rightarrow \mathbb{R}$ is strictly convex and differentiable, and $h_{i j}\left(w_{i} ; \cdot\right): \mathbb{R}^{m_{i}} \rightarrow \mathbb{R}$ is convex and differentiable. We denote by $r_{i}^{\sharp}$ the optimal solution to (2).

An independent public commission, called utility in the remaining of this paper, also tries to incorporate economic efficiency of the agents, but its most important priority is on fulfillment operating constraints at the steady-state such as $L(w) z+\ell_{w}(w)=0$, where, we set $z=\left[z_{i}\right]_{i \in N}$ and $w=\left[w_{i}\right]_{i \in\{0\} \cup N} \in W=W_{0} \times \prod_{i \in N} W_{i}$, and $w_{0} \in W_{0} \subset \mathbb{R}^{m_{w 0}}$ denotes an additional exogenous input for representing the steady-state constraints. The utility tries to determine the socially optimal solution as the optimal solution to the following problem.

$$
\min _{r} \sum_{i \in N} J_{i}\left(w_{i} ; r_{i}\right)
$$

subject to $h_{i j}\left(w_{i} ; r_{i}\right) \leq 0 \quad i \in N \quad j \in T_{i}$

$$
L(w) r+\ell_{w}(w)=0
$$

where $r=\left[r_{i}\right]_{i \in N} \in \mathbb{R}^{m}$ and $m=\sum_{i \in N} m_{i}$. The matrices $L(w)=\left[\begin{array}{llll}L_{1}(w) & L_{2}(w) & \cdots & L_{n}(w)\end{array}\right] \in \mathbb{R}^{\ell \times m}, L_{i}:$ $W \rightarrow \mathbb{R}^{\ell \times m_{i}}, i \in N$ and $\ell_{w}: W \rightarrow \mathbb{R}^{\ell}$ define equality constraints that the utility want to satisfy. We denote by $r_{i}^{*}$, $i \in N$ the optimal solution to (3).

Each agent $G_{i}$ is allowed to determine its own optimal setpoint through the optimization of (2). However, it is unlikely to hold the alignment of the distribute decision making $r_{i}^{\sharp}$ of each agent in (2) with the socially optimal solution $r_{i}^{*}$ to (3). In order to align the individual decision making of each agent with the socially optimal solution, we suppose that the utility is allowed to provide an additional price $p_{i} \in$ $\mathbb{R}^{m_{i}}, i \in N$, or, in other word, tax to the agent or subsidy from the community. Therefore, each agent $G_{i}$ participates to the community through the optimization of the following problem.

$$
\begin{array}{cl}
\min _{r_{i}} & J_{i}\left(w_{i} ; r_{i}\right)+p_{i}^{\mathrm{T}} r_{i} \\
\text { subject to } & h_{i j}\left(w_{i} ; r_{i}\right) \leq 0 \quad j \in T_{i}
\end{array}
$$

For each given $p_{i}$, we denote by $r_{i}^{b}\left(p_{i}\right)$ the optimal solution to (4).

We consider the feedback interactions between the agents $G_{i}, i \in N$ and the utility. The utility tries to determine and provide the price $p_{i}(t)$ in real-time, and the agent $G_{i}$ determines its own input $r_{i}(t)$ as the solution to (4) in realtime according to the provided price $p_{i}(t)$. The problem can be stated as follows (see also the following Fig. 1): Design a real-time pricing strategy of the utility that determines $p_{i}(t)$ by utilizing the amount of net imbalance $L(w) z(t)+\ell_{w}(w)$, and eventually realizes $r_{i}^{b}\left(p_{i}(t)\right) \rightarrow r_{i}^{*}$ for all $i \in N$.

\section{Standing Assumptions}

We list standing assumptions.

Assumption 1: For each $w \in W$, the matrix $L(w)$ has full row rank, i.e., $\operatorname{rank} L(w)=\ell$.

Assumption 1 implies that the representation of the equality constraints in (3c) includes no redundant constraint.

Assumption 2: For each $w \in W$, constraints in (3b) and (3c) satisfy Slater's constraint qualification condition.

Slater's constraint qualification condition implies that strong duality holds for the problem (3) [17] and the KKT (Karush-Kuhn-Tucker) conditions are necessary and sufficient condition for optimality.

Assumption 3: For each $w \in W$, there exists an optimal solution to (3).

Due to strict convexity of each $J_{i}$, the optimal solution to (3) is unique for each $w \in W$, if it exists.

Assumption 4: Let $w \in W$ be a given constant:

1) There exits an equilibrium point $x$ that satisfies

$$
0=f_{i}\left(x_{i}, g^{i}\left(x^{i}\right), w_{i}, r_{i}\right)
$$

for all $i \in N$, if and only if, $r$ satisfies (3c).

2) Let $r$ be any constant that satisfies (3c). The equilibrium point $x$ satisfies

$$
r_{i}=g_{i}\left(x_{i}, g^{i}\left(x^{i}\right), w_{i}\right)
$$

for all $i \in N$.

3) There exists a linearization of (1) around $(x, r)$

$$
\begin{aligned}
\delta \dot{x}_{i} & =a_{i}(w) \delta x_{i}+a^{i}(w) \delta x^{i}+b_{i}(w) \delta r_{i} \\
\delta z_{i} & =c_{i}(w) \delta x_{i}+c^{i}(w) \delta x^{i} \quad i \in N
\end{aligned}
$$

where $a_{i}(w)=\partial f_{i} / \partial x_{i}, a^{i}(w)=\partial\left(f_{i} g^{i}\right) / \partial x^{i}$, $b_{i}(w)=\partial f_{i} / \partial r_{i}, c_{i}(w)=\partial g_{i} / \partial x_{i}$ and $c^{i}(w)=$ $\partial\left(g_{i} g^{i}\right) / \partial x^{i}$, respectively. 
4) Define the matrices $A(w), B(w)$ and $C(w)$, by appropriately aligning the matrices in (6), which represent the entire linearized dynamics of (1), then the matrix $A(w)$ is Hurwitz.

We suppose that (1) represents a closed-loop system that consists of plant to be controlled and local feedback controller, which already has been designed. Assumption 4 requires that this closed-loop system has already been designed, and it realizes error free steady state tracking, $\lim _{t \rightarrow \infty} z_{i}(t)=r_{i}$, at least locally.

In application to the optimal power flow problem in Section IV, the problem satisfies Assumption 4, except item 4), i.e., the power flow model is not asymptotically stable. Specific comments to the power flow problem will be stated in Section IV. Assumption 4-1) may seem to be restrictive, but if one considers more relaxed case such as the condition holds for any given constants $w \in W$ and $r$, the results in this paper still remain valid.

\section{Summaries of the PRICING StRategy AND STABILITY ANALYSIS}

This section summarizes the results on the real-time pricing strategy and stability analysis.

\section{A. Real-time Pricing Strategy}

We start with investigating steady-state optimality and provide a pricing strategy of the utility that aligns the distributed decision making $r_{i}^{b}$ of the agent in (4) with the socially optimal solution $r_{i}^{*}$ to (3).

Let us consider the KKT conditions of (3).

$$
\begin{aligned}
\frac{\partial J_{i}\left(w_{i} ; r_{i}\right)}{\partial r_{i}}+L_{i}^{\mathrm{T}}(w) \lambda+\sum_{j \in T_{i}} \frac{\partial h_{i j}\left(w_{i} ; r_{i}\right)}{\partial r_{i}} \mu_{i j} & =0 \\
h_{i j}\left(w_{i} ; r_{i}\right) \leq 0 \quad \mu_{i j} \geq 0 \quad h_{i j}\left(w_{i} ; r_{i}\right) \times \mu_{i j} & =0 \\
i \in N \quad j & \in T_{i} \\
L(w) r+\ell_{w}(w) & =0
\end{aligned}
$$

The following Lemma 1 states that, at least in steady-state, the utility can choose the optimal price $p_{i}^{*}$ that realizes $r_{i}^{b}\left(p_{i}^{*}\right)=r_{i}^{*}$ for all $i \in N$.

Lemma 1: Let $w \in W$ be given, and let us denote the optimal solution to (3) or, equivalently, variables that satisfy the KKT conditions in (7) as $r_{i}^{*}, \mu_{i j}^{*}, i \in N, j \in T_{i}$ and $\lambda^{*}$. If the utility provides the price, $p_{i}$, according to the pricing strategy $p_{i}=L_{i}^{\mathrm{T}}(w) \lambda \in \mathbb{R}^{m_{i}}, i \in N$, then we have $r_{i}^{\mathrm{b}}\left(p_{i}^{*}\right)=$ $r_{i}^{\mathrm{b}}\left(L_{i}^{\mathrm{T}}(w) \lambda^{*}\right)=r_{i}^{*}$.

The utility need to solve the optimization problem (3) or the KKT conditions in (7) to determine the optimal price $p_{i}^{*}=L_{i}^{\mathrm{T}}(w) \lambda^{*}$. However, the problem becomes very large, since we may have a huge number of agents. It may be difficult to solve (3) or (7) and provide its solution $p_{i}^{*}$ in real-time.

We investigate a real-time pricing strategy of the utility that utilizes the amount of net imbalance of the constraints over the network. Let us start with the dual problem of (3)

$$
\max _{\lambda} \min _{\substack{r \\ h_{i j}\left(w_{i} ; r_{i}\right) \leq 0 \\ i \in N}} \sum_{i \in T_{i}} J_{i}\left(w_{i} ; r_{i}\right)+\lambda^{\mathrm{T}}\left(L(w) r+\ell_{w}(w)\right)
$$

If we consider only the optimality in steady-state, as similar to the case of Lemma 1, $r_{i}$ will be determined as $r_{i}^{b}$ by each agent $G_{i}$. By substituting this, we have

$$
\max _{\lambda} \sum_{i \in N} J_{i}\left(w_{i} ; r_{i}^{b}\right)+\lambda^{\mathrm{T}}\left(L(w) r^{\mathrm{b}}+\ell_{w}(w)\right)
$$

where $r^{b}=\left[r_{i}^{b}\right]_{i \in N}$. We apply the gradient method to this maximization problem, then we have

$$
\frac{d \lambda}{d \tau}=\epsilon\left(L(w) r^{b}+\ell_{w}(w)\right) \quad \epsilon>0
$$

We replace $r^{b}$ by its corresponding output $z(t)$ in the above equation and combine the result in Lemma 1. Then, a heuristic real-time pricing strategy of the utility is given as

$$
\begin{aligned}
\dot{\lambda}(t) & =\epsilon\left(L(w) z(t)+\ell_{w}(w)\right) \quad \epsilon>0 \\
p_{i}(t) & =L_{i}^{\mathrm{T}}(w) \lambda(t) \quad i \in N
\end{aligned}
$$

Fig. 1 shows a schematic block diagram of the proposed closed-loop system that equipped with a gradient based pricing strategy (8) and distributed optimization (4) of each agent $G_{i}$, where we note that, in the proposed closed-loop system, no-one need to solve a large scale optimization problem nor consider iterative calculations to determine the price. In addition, to determine the price, the utility does not need to know any specific properties, like functions $f_{i}$ or $J_{i}$ for example, of the agent $G_{i}$.

\section{B. Stability Analysis}

It has been shown in [15] that, by investigating the local dynamics around the equilibrium point, the resulting closedloop system in Fig. 1 is stable at least locally for sufficiently small $\epsilon>0$.

Definition 1: Let $w \in W$ be a given constant. A triple $(x, \lambda, r)$ is said to be an equilibrium point of the closed-loop system in Fig. 1 corresponding to $w$, if

$$
\begin{aligned}
0 & =f_{i}\left(x_{i}, g^{i}\left(x^{i}\right), w_{i}, r_{i}\right) \\
0 & =\epsilon\left(L(w) g(x, w)+\ell_{w}(w)\right) \\
r_{i} & =g_{i}\left(x_{i}, g^{i}\left(x^{i}\right), w_{i}\right) \\
r_{i} & =r_{i}^{b}\left(L_{i}^{\mathrm{T}}(w) \lambda\right)
\end{aligned}
$$

are held for all $i \in N$, where $g(x, w)=$ $\left[g_{i}\left(x_{i}, g^{i}\left(x^{i}\right), w_{i}\right)\right]_{i \in N}$.

Note that $x$ and $\lambda$ is a part of the state, but $r$ is not. The following lemma is a conclusion from Assumptions 3 and 4-1), 2),

Lemma 2: For each given constant $w \in W$, the closedloop system in Fig. 1 has an equilibrium point $(x, \lambda, r)$ that satisfies (5).

Let $r^{*}$ be an optimal solution to (3) for a given $w \in W$. The proof of Lemma 2 shows that there exist $x^{*}$ and $\lambda^{*}$ such that the triple $\left(x^{*}, \lambda^{*}, r^{*}\right)$ can be an equilibrium, as well as, it satisfies (5).

Let $w \in W$ be given, and let us denote by $r_{i}^{*}, \mu_{i j}^{*}$, $i \in N, j \in T_{i}$ and $\lambda^{*}$ the corresponding optimal solution to (3) or, equivalently, the variables which satisfy the KKT conditions in (7). We set $p_{i}^{*}=L_{i}^{\mathrm{T}}(w) \lambda^{*}, i \in N$ and 


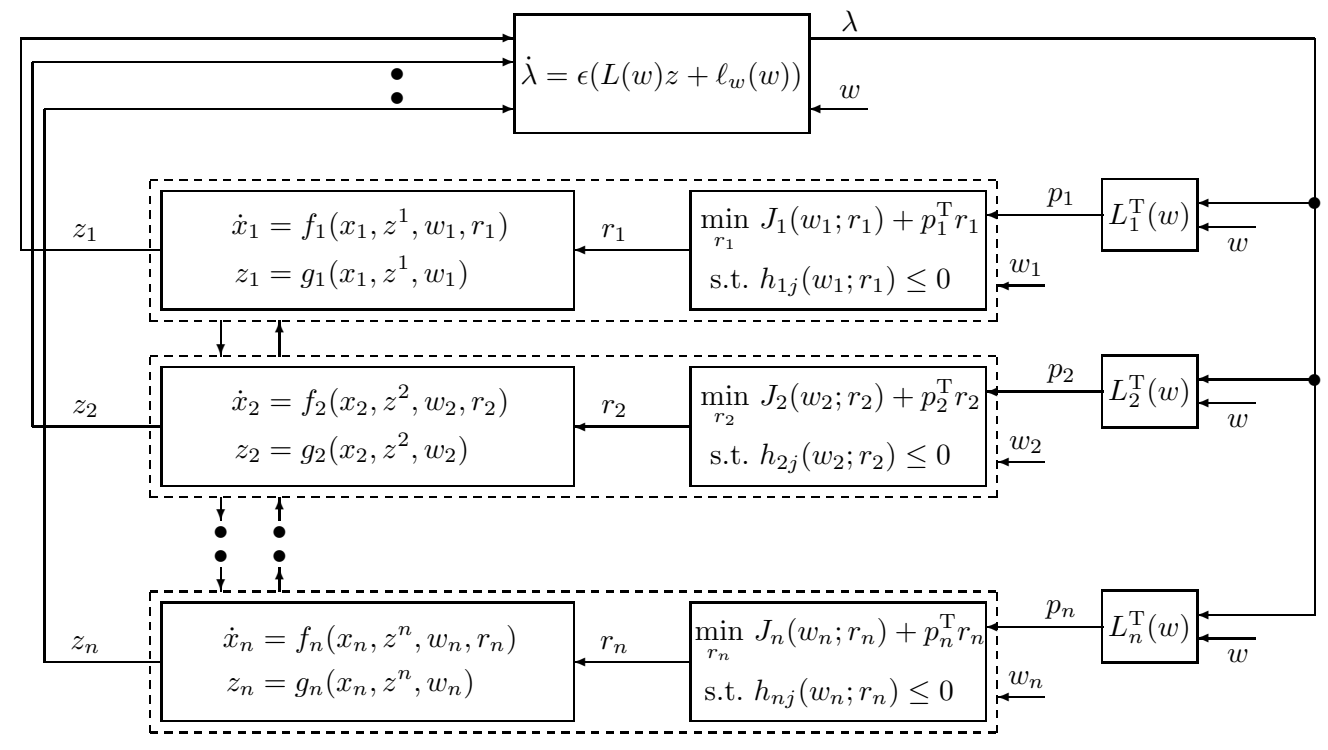

Fig. 1. A schematic block diagram of the distributed optimization and integrate pricing strategy mechanisms.

consider the optimal solution $r_{i}^{b}\left(p_{i}^{*}\right)$ to (4). In order to capture the local behavior of the optimization problem in (4), we need to consider the gradient $E_{i}(w)=-\left(\partial r_{i} / \partial p_{i}\right)$ that satisfies $r_{i}^{\mathrm{b}}\left(p_{i}^{*}+\delta p_{i}\right)=r_{i}^{\mathrm{b}}\left(p_{i}^{*}\right)-E_{i}(w) \delta p_{i}+\mathcal{O}\left(\left\|\delta p_{i}\right\|^{2}\right)$ for a small deviation $\delta p_{i}$ from $p_{i}^{*}$. Under the appropriate technical assumption, an explicit procedure to calculate the gradient $E_{i}(w)$ has been derived in [15]. We set $E(w)=$ block $\operatorname{diag}\left(E_{1}(w), E_{2}(w), \ldots, E_{n}(w)\right)$, and the stability result can be summarized as in the following theorem.

Theorem 1: Let $w \in W$ be a given constant, and let a triple $(x, \lambda, r)$ be an equilibrium point in Lemma 2. Suppose that the matrix $L(w) E(w) L^{\mathrm{T}}(w)$ is non-singular. The triple $(x, \lambda, r)$ is a stable equilibrium point of the closed-loop system in Fig. 1 at least locally, if $\epsilon>0$ in (8) is sufficiently small.

The matrix $L(w)$ has full row $\operatorname{rank}, \operatorname{rank} L(w)=\ell$, but each matrix $E_{i}(w)$ is only positive semi-definite, $E_{i}(w) \geq 0$, and there is a possibility that $L(w) E(w) L^{\mathrm{T}}(w)$ becomes singular. If the matrix $L(w) E(w) L^{\mathrm{T}}(w)$ is singular, the linearized dynamics of the closed-loop system in Fig. 1 necessarily has a zero-eigenvalue and is not stable, thus $L(w) E(w) L^{\mathrm{T}}(w)>0$ is a necessary condition for stability of the linearized system.

The structural stability condition in Theorem 1 is preferred, since the network may have huge number of agents, and a numerical approach for stability analysis becomes difficult. Theorem 1 also shows that an integral action, $\lambda=((\epsilon I) / s)\left(L(w) z+\ell_{w}(w)\right)$, to determine the price can stabilize the resulting closed-loop system. This inspires that integral plus phase-lead compensation

$$
\lambda=\frac{\epsilon}{s} \frac{\omega_{L}}{\omega_{\ell}} \frac{s+\omega_{\ell}}{s+\omega_{L}} I\left(L(w) z+\ell_{w}(w)\right) \quad \omega_{L}>\omega_{\ell}>0
$$

may be useful and stabilize the closed-loop system. If one utilizes phase-lead action, it may allow to use larger $\epsilon>0$ and result in a quicker response of the closed-loop system. In a large network, communication delays could arise another issue, and an effect of time-delays due to communication networks in the proposed real-time pricing strategy is studied in [18]. On the other hand, if one considers a small network, like a network so-called micro-grid, a numerical computation for stability analysis may be acceptable. In this case, stability conditions in LMIs can be obtained without introducing the gradient of the optimization problem [19].

\section{Application Case Study: Leading to Optimal POWER FLOW}

This section considers the New England 39-bus test system [16]. The network topology, generators and loads are depicted in Fig. 2. Detailed descriptions of power flow analysis and DC power flow model can be found in standard text books and related literature [20], [21], [3], [22].

We suppose that the network has $n=n_{\ell}+n_{g}$ nodes, where $n_{g}$ denotes a number of nodes that equipped with generators and $n_{\ell}$ denotes a number of nodes without generators ${ }^{1}$. We also denote $N_{\ell}=\left\{1,2, \ldots, n_{\ell}\right\}, N_{g}=\left\{n_{\ell}+1, n_{\ell}+\right.$ $2, \ldots, n\}$ and $N=N_{\ell} \cup N_{g}$.

Let $p_{\ell i j}$ and $p_{g i j}$ denote the outgoing power flow from node $i \in N$ to neighboring node $j \in N_{i}$. Let us set $p_{\ell i}=$ $\sum_{j \in N_{i}} p_{\ell i j}, i \in N_{\ell}, p_{g k}=\sum_{j \in N_{k}} p_{g k j}, k \in N_{g}, p_{\ell}=$ $\left[p_{\ell i}\right]_{i \in N_{\ell}}$ and $p_{g}=\left[p_{g i}\right]_{i \in N_{g}}$, respectively. We use the DC power flow model to determine the power flow in the network, and with the DC power flow model, the power flow in the network is determined as

$$
\left[\begin{array}{l}
p_{\ell} \\
p_{g}
\end{array}\right]=\left[\begin{array}{ll}
M_{11} & M_{12} \\
M_{21} & M_{22}
\end{array}\right]\left[\begin{array}{l}
\theta_{\ell} \\
\theta_{g}
\end{array}\right]
$$

where, we set $\theta_{\ell}=\left[\theta_{\ell i}\right]_{i \in N_{\ell}}, \theta_{g}=\left[\theta_{g i}\right]_{i \in N_{g}}$, and each $\theta_{\ell i}$ or $\theta_{g i}$ denotes a voltage phase angel at the node $i$. The

\footnotetext{
${ }^{1}$ If one looks the New England 39-bus test system in Fig. 2, it has $n=39$ nodes, $n_{g}=10$ generator nodes and $n_{\ell}=n-n_{g}=29$ consumption/hub nodes, respectively.
} 


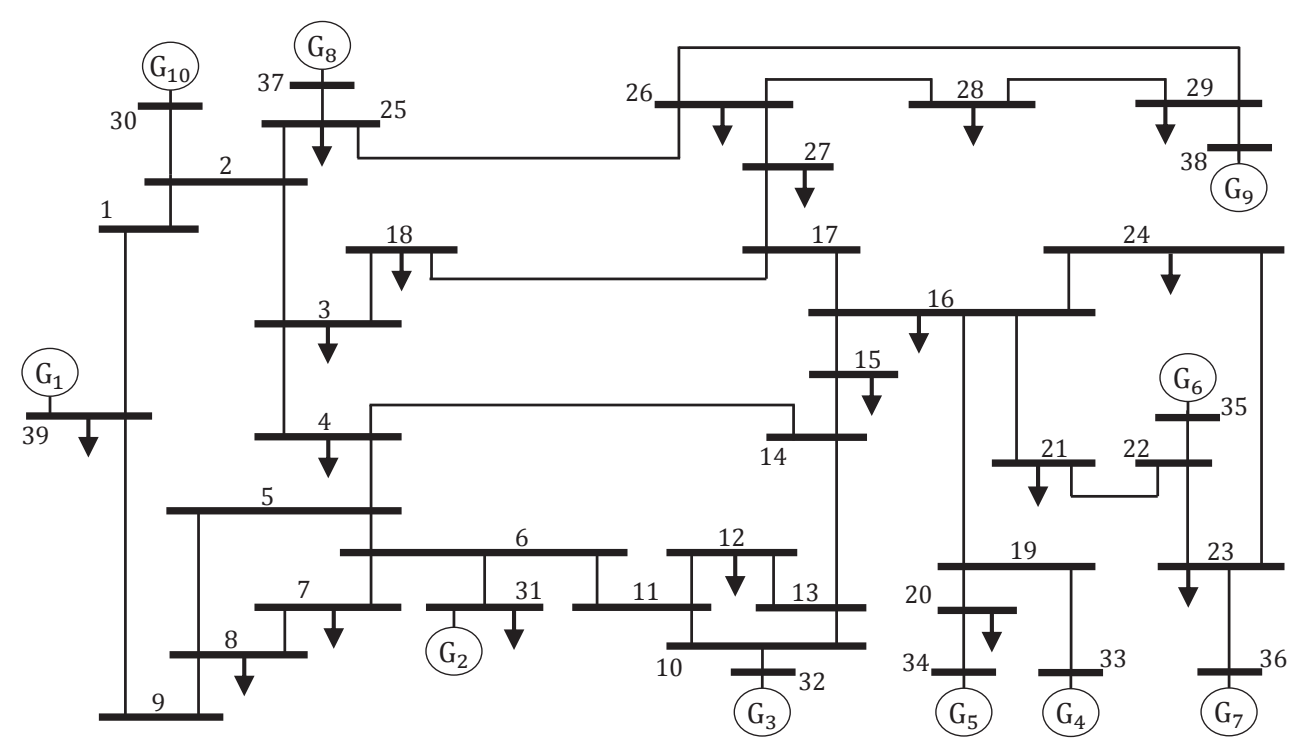

Fig. 2. The New England 39-bus test system.

constant matrix $M=\left[M_{i j}\right]_{i, j \in\{1,2\}}$ is determined from the line susceptance parameters of the network, and it is symmetric $M=M^{\mathrm{T}}$ and singular with rank deficiency one. This physically reflects only the relative voltage phase angles determine the power flow. We consider quadratic functions $J_{i}\left(r_{i}\right)=(1 / 2) c_{g i} r_{i}^{2}+b_{g i} r_{i}, i \in N_{g}$ to represent the production cost of steady power generation $r_{i}$ at each generator node.

The optimal DC power flow problem, without considering any dynamics of generators and loads, investigates economic efficiency of the steady power generation and determines the steady-state power flow in the network for a given amount of consumption at each node as the solution to the following optimization problem.

$$
\begin{aligned}
\min _{\theta_{\ell}, \theta_{g}, r} & \sum_{i \in N_{g}} J_{i}\left(r_{i}\right) \\
\text { subject to } & {\left[\begin{array}{ll}
M_{11} & M_{12} \\
M_{21} & M_{22}
\end{array}\right]\left[\begin{array}{l}
\theta_{\ell} \\
\theta_{g}
\end{array}\right]=\left[\begin{array}{l}
0 \\
r
\end{array}\right]-\left[\begin{array}{l}
w_{\ell} \\
w_{g}
\end{array}\right] }
\end{aligned}
$$

where, we set $r=\left[r_{i}\right]_{i \in N_{g}}, w_{\ell}=\left[w_{\ell i}\right]_{i \in N_{\ell}}, w_{g}=$ $\left[w_{g i}\right]_{i \in N_{g}}$, and each $w_{\ell i}$ or $w_{g i}$ represents the amount of consumption at the consumption/hub or generator node $i^{2}$, and $r_{i}$ represents amount of power injection or steady-state set-point at the generator node $i \in N_{g}$. The matrix $M$ in (10b) has rank $n-1$ with the kernel space $\operatorname{span}\left\{\mathbb{1}_{n}\right\}$. Since $M=M^{\mathrm{T}}$, we have $\operatorname{span}\left\{\mathbb{1}_{n}\right\} \perp \operatorname{Im} M$. For any given $w=\left[\begin{array}{ll}w_{\ell}^{\mathrm{T}} & w_{g}^{\mathrm{T}}\end{array}\right]^{\mathrm{T}}$, the constraints in (10b) can be feasible,

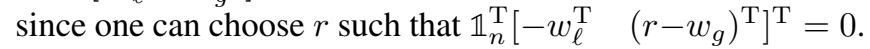

Instead to solve a large optimization problem in (10), as well as, incorporating dynamical interactions between generators and loads, we apply the real-time pricing strategy and distributed decision makings summarized in Section III. We

${ }^{2}$ If one looks the New England 39-bus test system in Fig. 2, the node 31 and 39 have power consumption as well as generators. start with considering the following standard swing equation as a dynamic model of consumption/hub node $i \in N_{\ell}$.

$$
2 H_{\ell i} \ddot{\theta}_{\ell i}(t)=-k_{\ell i} \dot{\theta}_{\ell i}-p_{\ell i}(t)-w_{\ell i}(t)
$$

where $p_{\ell i}$ represents the outgoing net power flow from node $i$ to neighboring nodes $j \in N_{i}$. We also have the following state space representation.

$$
\begin{aligned}
{\left[\begin{array}{c}
\dot{\theta}_{\ell i} \\
\dot{\omega}_{\ell i}
\end{array}\right]=} & {\left[\begin{array}{cc}
0 & 1 \\
0 & -k_{\ell i} /\left(2 H_{\ell i}\right)
\end{array}\right]\left[\begin{array}{c}
\theta_{\ell i} \\
\omega_{\ell i}
\end{array}\right] } \\
& +\left[\begin{array}{c}
0 \\
1 /\left(2 H_{\ell i}\right)
\end{array}\right]\left(-p_{\ell i}\right)-\left[\begin{array}{c}
0 \\
1 /\left(2 H_{\ell i}\right)
\end{array}\right] w_{\ell i}
\end{aligned}
$$

where $\omega_{\ell i}=\dot{\theta}_{\ell i}$, and we note here that $-p_{\ell i}$ represents the incoming flow into the node $i$.

As a dynamic model of generator node $i \in N_{g}$, we consider the following swing equation.

$$
\begin{aligned}
2 H_{g i} \ddot{\theta}_{g i}(t) & =u_{i}(t)+r_{i}(t)-\left(w_{g i}(t)+p_{g i}(t)\right) \\
u_{i}(t) & =-k_{g i} \dot{\theta}_{g i}(t)
\end{aligned}
$$

where $u_{i}$ is a local feedback control, and $p_{g i}$ represents the outgoing net power flow form generator node $i$ to neighboring nodes $j \in N_{i}{ }^{3}$. We can consider the state space representation

$$
\begin{aligned}
{\left[\begin{array}{c}
\dot{\theta}_{g i} \\
\dot{\omega}_{g i}
\end{array}\right]=} & {\left[\begin{array}{cc}
0 & 1 \\
0 & -k_{g i} /\left(2 H_{g i}\right)
\end{array}\right]\left[\begin{array}{c}
\theta_{g i} \\
\omega_{g i}
\end{array}\right] } \\
& +\left[\begin{array}{c}
0 \\
1 /\left(2 H_{g i}\right)
\end{array}\right] r_{i}-\left[\begin{array}{c}
0 \\
1 /\left(2 H_{g i}\right)
\end{array}\right]\left(w_{g i}+p_{g i}\right)
\end{aligned}
$$

where $\omega_{g i}=\dot{\theta}_{g i}$. We also set the output equation as

$$
z_{i}=p_{g i}+w_{g i} \quad i \in N_{g}
$$

\footnotetext{
${ }^{3}$ As the model of generator, one can consider turbine and governor dynamics with the swing equation in (12) [20], [21]. We consider a simple generator model by omitting turbine and governor dynamics.
} 
The value of $z_{i}$ represents the net outgoing power flow from the node $i$, i.e., the amount of power generation at node $i \in N_{g}$, thus it should be tracked to the set-point $r_{i}$.

By using appropriate matrices $A, B$ and $C=$ [ $\left.\begin{array}{lll}M_{21} & M_{22} & 0\end{array}\right]$, the entire dynamics in (11) and (13) is represented in the form

$$
\begin{aligned}
& \dot{x}=\left(A-B\left[\begin{array}{ll}
M & 0
\end{array}\right]\right) x-B w+B\left[\begin{array}{l}
0 \\
r
\end{array}\right] \\
& z=C x+w_{g}
\end{aligned}
$$

where $x=\left[\begin{array}{llll}\theta_{\ell}^{\mathrm{T}} & \theta_{g}^{\mathrm{T}} & \omega_{\ell}^{\mathrm{T}} & \omega_{g}^{\mathrm{T}}\end{array}\right]^{\mathrm{T}}, \omega_{\ell}=\left[\begin{array}{l}\omega_{\ell i}\end{array}\right]_{i \in N_{\ell}}$, $\omega_{g}=\left[\omega_{g i}\right]_{i \in N_{g}}, w=\left[w_{\ell}^{\mathrm{T}} \quad w_{g}^{\mathrm{T}}\right]^{\mathrm{T}}$ and $z=\left[z_{i}\right]_{i \in N_{g}}$. We also consider the extended output equation, which makes the subsequent presentation simple

$$
\hat{z}=\left[\begin{array}{c}
z_{\ell} \\
z
\end{array}\right]=\left[\begin{array}{c}
p_{\ell}+w_{\ell} \\
z
\end{array}\right]=\hat{C} x+w
$$

where $p_{\ell}=\left[p_{\ell i}\right]_{i \in N_{\ell}}$ and $\hat{C}=\left[\begin{array}{ll}M & 0\end{array}\right]$. The output $\left(z_{\ell}\right)_{i}$, $i \in N_{\ell}$ represents the net outgoing power flow from the consumption/hub node $i$. We need to have $\left(z_{\ell}\right)_{i}=0$ at steady state, i.e., the incoming and outgoing net power flow at the consumption/hub node should be balanced.

Let $r^{\natural}$ denote the optimal solution to (10). The optimal DC power flow problem in (10) gives the optimal steady-state set-point $r$ to each generator $i \in N_{g}$ as its optimal solution $r^{\natural}$, but we do not solve the large optimization problem (10). In our real-time pricing and distributed decision making setting, the interaction model of dynamic agents in (1) is given as (14a) and (14b), and the optimization problem that corresponds to (3) and defines social optimality is given as

$$
\begin{array}{cl}
\min _{r} & \sum_{i \in N_{g}} J_{i}\left(r_{i}\right) \\
\text { subject to } & \mathbb{1}_{n_{g}}^{\mathrm{T}} r-\mathbb{1}_{n}^{\mathrm{T}} w=0
\end{array}
$$

Let $r^{*}$ denote the optimal solution to (15). We actually have $r^{\natural}=r^{*}$, as we will see in the followings.

Lemma 3: Let $w \in W$ be given. There exists an equilibrium point $x$ to (14a), if and only if, $r$ satisfies (15b). The equilibrium point $x$ also satisfies $\left[\begin{array}{ll}0^{\mathrm{T}} & r^{\mathrm{T}}\end{array}\right]^{\mathrm{T}}=\hat{C} x+w$.

We immediately have the following corollary.

Corollary 1: The dynamic network of loads and generators in (14a), (14b) and the optimization problem (15) satisfy Assumptions 4-1) and 2).

Lemma 4: Let $w \in W$ be given, and let $\left(\theta_{\ell}^{\natural}, \theta_{g}^{\natural}, r^{\natural}\right)$ and $r^{*}$ be the optimal solutions to (10) and (15), respectively. We have $r^{\natural}=r^{*}$.

We apply the proposed real-time pricing and distributed decision makings methodology to the optimal DC power flow problem. Each generator solves

$$
\min _{r_{i}} J_{i}\left(r_{i}\right)+p_{i}^{\mathrm{T}} r_{i} \quad i \in N_{g}
$$

in real-time and decides its own reference input $r_{i}^{b}\left(p_{i}(t)\right)$ as a solution to (16), where $p_{i}$ is the price provided by the utility according to the gradient based pricing strategy

$\dot{\lambda}(t)=\epsilon\left(\mathbb{1}_{n_{g}}^{\mathrm{T}} z(t)-\mathbb{1}_{n}^{\mathrm{T}} w(t)\right) \quad p_{i}(t)=\lambda(t) \quad i \in N_{g}$
From Theorem 1, we could conclude stability of the resulting closed-loop system, which consists of (14a), (14b), (16) and (17), provided that (14) satisfies Assumption 44), i.e., it is asymptotically stable. However, the power flow network (14) may not be designed as asymptotically stable system. This physically reflects the fact that only the relative voltage phase angles determine the power flow, and the system necessarily has zero eigenvalue with eigenvector $\left[\begin{array}{ll}\mathbb{1}^{\mathrm{T}} & 0^{\mathrm{T}}\end{array}\right]^{\mathrm{T}}$. Although the power flow network (14) is not asymptotically stable, stability of the resulting closed-loop system can still be concluded by introducing slack node and considering simple coordinate transformation. For the shake of simplicity, we suppose that $i=1 \in N_{\ell}$ is the slack node. Since we are only interested in the relative voltage phase angle, by introducing an appropriate matrix $T \in \mathbb{R}^{2 n \times 2 n}$, we consider the coordinate transformation $\left[\begin{array}{ll}\theta_{\ell 1} & \xi^{\mathrm{T}}\end{array}\right]^{\mathrm{T}}=T x$, where, we set $\xi=\left[\left(\left[\delta_{i}\right]_{i \in N \backslash\{1\}}\right)^{\mathrm{T}} \quad \omega_{\ell}^{\mathrm{T}} \quad \omega_{g}^{\mathrm{T}}\right]^{\mathrm{T}}$, and $\delta_{i}$ is defined as $\theta_{\ell i}-\theta_{\ell 1}$ for $i \in N_{\ell} \backslash\{1\}$ and $\theta_{g j}-\theta_{\ell 1}$ for $j \in N_{g}$. By utilizing the facts that the inverse transformation is represented by the matrix

$$
T^{-1}=\operatorname{diag}\left(T_{11}^{-1}, I_{n}\right) \quad T_{11}^{-1}=\left[\begin{array}{cc}
\mathbb{1}_{n} & 0 \\
& I_{n-1}
\end{array}\right]
$$

and $M \mathbb{1}_{n}=0$, the system (14a) and (14b) is transformed into the form

$$
\begin{aligned}
\dot{\theta}_{\ell 1} & =\omega_{\ell 1} \\
\dot{\xi} & =\tilde{A} \xi+\tilde{B} w+\tilde{B}\left[\begin{array}{l}
0 \\
r
\end{array}\right] \\
z & =\tilde{C} \xi+w_{g}
\end{aligned}
$$

From this, we can see that the voltage phase angle of the slack node does not affect the amount of power generation.

We can summarize the convergence property of the closedloop system as in the following corollary.

Corollary 2: Suppose that (18b) satisfies Assumption 44), i.e., the matrix $\tilde{A}$ is Hurwitz, and $\epsilon>0$ in (17) is sufficiently small. Let $w$ be a given constant. The closed-loop system that consists of (14a), (14b), (16) and (17) satisfies $r_{i}^{b}(p(t)) \rightarrow r_{i}^{*}$ and $z_{i}(t) \rightarrow r_{i}^{*}$ as $t \rightarrow \infty$.

The following two subsections consider the New England 39-bus test system and present simulation results. The details of the New England 39-bus test system such as the bus parameters that define the matrix $M$ can be found in the literature (see [16], for example). We take the values of parameters $c_{g i}$ and $b_{g i}, i \in N_{g}$ from [7], [3], and we use $H_{\ell i}=5, k_{\ell i}=10, i \in N_{\ell}, H_{g j}=5$ and $k_{g j}=10, j \in N_{g}$ in the following simulations.

\section{A. Realizing Optimal Power Flow}

In this simulation, we suppose that, at time instance $5[\mathrm{~s}]$, the load demands increase or decrease as $w_{\ell 3}, w_{\ell 4} \rightarrow+20 \%$, $w_{\ell 26}, w_{\ell 27} \rightarrow-20 \%$ and $w_{\ell 28}, w_{\ell 29} \rightarrow+40 \%$, respectively (the load demands on the other nodes are unchanged).

Figs. 3(a) and 3(b) show samples of the resulting time response on consumption/hub nodes. The dashed-dotted line indicates the load demand $w_{\ell i}$ and solid line indicates $-z_{\ell i}$ 


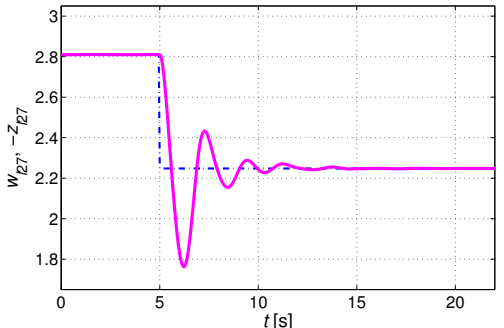

(a) Consumption node 27: $w_{\ell 27}$ and $-z_{\ell 27}$

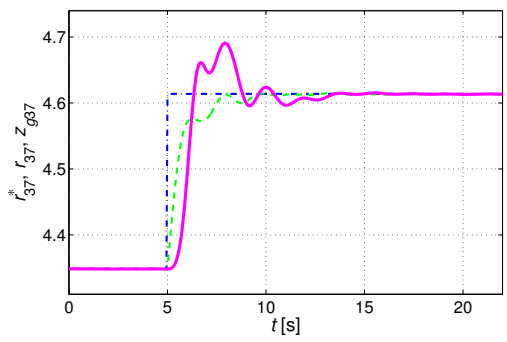

(d) Generator node 37: $r_{37}^{*}, r_{37}^{b}$ and $z_{37}$

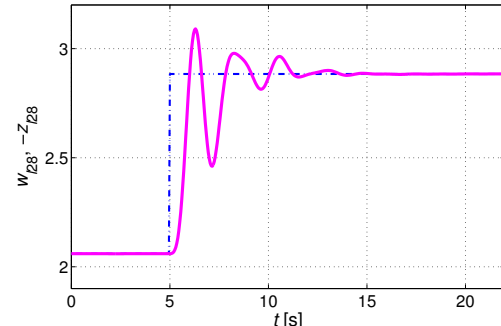

(b) Consumption node 28: $w_{\ell 28}$ and $-z_{\ell 28}$

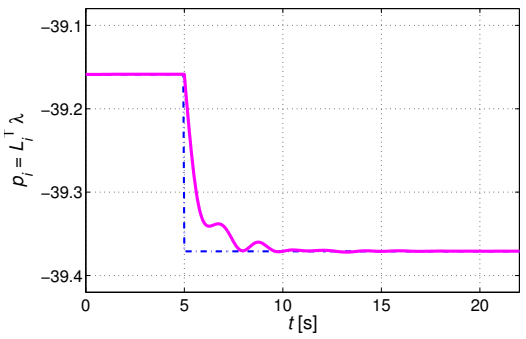

(e) Price, $p_{i}=L_{i}^{\mathrm{T}} \lambda=\lambda$, determined by the pricing strategy (8).

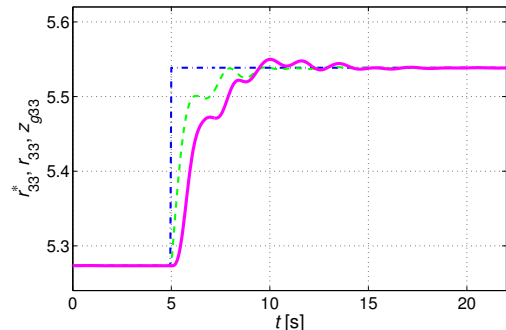

(c) Generator node 33: $r_{33}^{*}, r_{33}^{b}$ and $z_{33}$

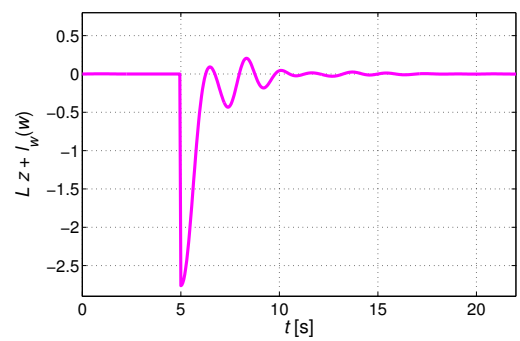

(f) A time history of $L(w) z+\ell_{w}(w)$ that indicates supply/demand balance.

Fig. 3. Sample time responses of the New England 39-bus test system.

(the net incoming power flow into the node $i$ ). We can see that, at the steady state, the outgoing power flow $w_{\ell i}$ and incoming power flow $-z_{\ell i}$ are balanced.

Figs. 3(c) and 3(d) show samples of the resulting time response on generator nodes. The dashed-dotted line indicates the solution $r_{i}^{\natural}=r_{i}^{*}$ to the optimal DC power flow problem in (10) or (15) (see Lemma 4). The dashed line indicates the reference input $r_{i}^{b}\left(p_{i}\right)$ generated as the solution to the distributed optimization problem in (16) according to the provided price $p_{i}(t)$. At the steady state, we can see that $r_{i}^{*}$ and $r_{i}^{b}\left(p_{i}\right)$ become identical each other, and the amount of power generation $z_{i}$ indicated by the solid line converges to the optimal value $r_{i}^{*}$.

Fig. 3(e) shows the resulting time response of price $p_{i}=\lambda$ determined by the utility according to the gradient based realtime pricing strategy (17). The dashed-dotted line indicates the value of optimal price $p_{i}^{*}=\lambda^{*}$, and we can see that $p_{i}$ converges to $p_{i}^{*}$. Fig. 3(f) shows the resulting time response of $L(w) z(t)+\ell_{w}(w)=\mathbb{1}_{n_{q}}^{\mathrm{T}} z(t)-\mathbb{1}_{n}^{\mathrm{T}} w(t)$, and we can see that the equality constraint, i.e., the net load and demand balance, is fulfilled in the steady-state. The simulation result shows that the proposed real-time pricing strategy and the distributed decision makings by each agent can lead the network to the optimal operating condition.

\section{B. Reducing the Effect of Unknown Renewable}

This simulation supposes that the network is equipped with window power generators, and we will see that the proposed real-time pricing and distributed decision makings methodology could reduce the effect of unknown renewable. We suppose that the node 17 is equipped with the window power generators, and Fig. 4(a) shows sample time history of the amount of power generation from the window power generators.
We apply the real-time pricing and distributed decision makings methodology in (16) and (17), and Figs. 4(b) and 4(c) show samples of the resulting time response of $\omega_{\ell i}$, which indicates frequency deviation form 50 or $60[\mathrm{~Hz}]$. In each figure, the dashed-dotted line indicates the frequency deviation without set-point control, and, since the set-point signal is fixed at each generator, the effect of window power generation at the node 17 directory appears as the frequency deviation at each node. On the other hand, the solid line indicates the frequency deviation with the price based set-point control. We can see that the effect of unknown renewable could be reduced.

\section{CONCLUSIONS}

Motivated by the control problems of distributed energy supply/demand networks, this paper investigated the optimal regulation problem with steady-state constraints under distributed decision makings, where each agent is allowed to determine its own optimal set-point according to an individual profit. On the other hand, the utility, which corresponds to an independent public commission, tries to realize a socially optimal solution that fulfills steady-state power balance equality constraints. In order to align the individual decision making of each agent with the socially optimal solution, the utility provides additional price, which conceptually represents tax or subsidy for the agent. This paper investigated application case studies of the proposed real-time pricing strategy and distributed decision makings methodology. By using the New England 39-bus test system, we considered the optimal power flow problem with the DC power flow model. The simulation results illustrated the effectiveness of the proposed method and validated the stability analysis procedure. 


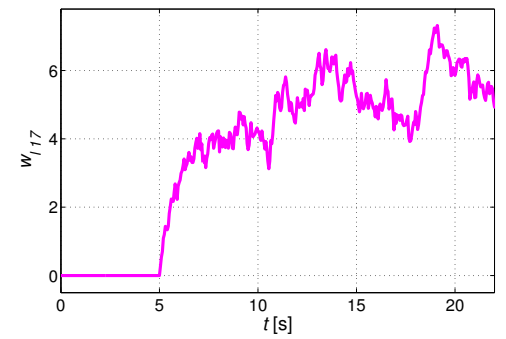

(a) A sample time history of the window power generation at node 17 . This sample time history is randomly generated.

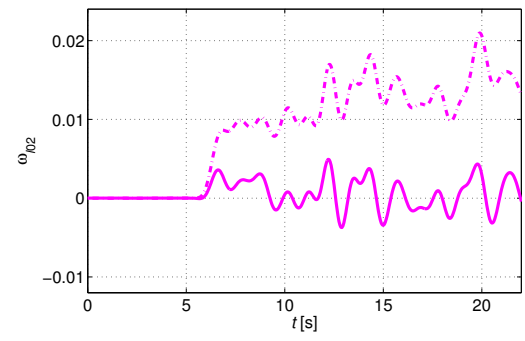

(b) Hub node $2, \omega_{\ell 2}$

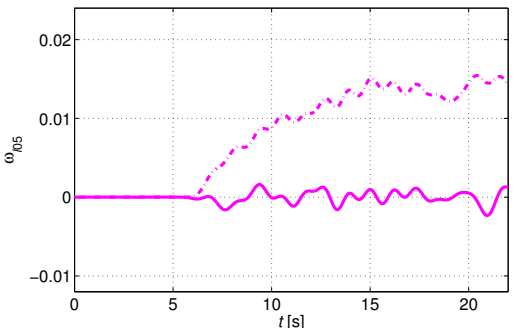

(c) Hub node $5, \omega_{\ell 5}$

Fig. 4. Sample time responses of frequency deviation due to the effect of unknown renewable.

\section{REFERENCES}

[1] A. Jokić, M. Lazar, and P. P. J. van den Bosch, "On constrained steadystate regulation: Dynamic KKT controllers," IEEE Transactions on Automatic Control, vol. 54, no. 9, pp. 2250-2254, 2009.

[2] D. DeHaan and M. Guay, "Extremum-seeking control of stateconstrained nonlinear systems," Automatica, vol. 41, no. 9, pp. 1567 - 1574, 2005.

[3] A. Jokić, M. Lazar, and P. van den Bosch, "Real-time control of power systems using nodal prices," International Journal of Electrical Power and Energy Systems, vol. 31, no. 9, pp. 522-530, 2009.

[4] K. Tsumura, H. Yamamoto, and D. Kiribuchi, "Generalization of Uzawa algorithm and its application," in the 13th SICE Annual Conference on Control Systems, 2013, pp. 8D3-6, in Japanese.

[5] A. W. Berger and F. C. Schweppe, "Real time pricing to assist in load frequency control," IEEE Transactions on Power Systems, vol. 4 no. 3, pp. 920-926, 1989.

[6] C. Silva, B. F. Wollenberg, and C. Z. Zheng, "Application of mechanism design to electric power markets (republished)," IEEE Transactions on Power Systems, vol. 16, no. 4, pp. 862-869, 2001.

[7] F. L. Alvarado et al., "Stability analysis of interconnected power systems coupled with market dynamics," IEEE Transactions on Power Systems, vol. 16, no. 4, pp. 695-701, 2001.

[8] N. Li, L. Chen, and S. H. Low, "Optimal demand response based on utility maximization in power networks," in Proceedings of the 2011 IEEE Power and Energy Society General Meeting, 2011, pp. 1 -8.

[9] Y. Okajima et al., "A dynamic mechanism for LQG power networks with random type parameters and pricing delay," in Proceedings of the 52nd IEEE Conference on Decision and Control, Florence, Italy, 2013, pp. 2384-2390.

[10] T. Murao et al., "Dynamic balanced integration mechanism for LQG power networks with independent types," in Proceedings of the 53rd IEEE Conference on Decision and Control, Los Angeles, 2014, pp. 1395-1402.

[11] A. Kiani and A. Annaswamy, "Wholesale energy market in a smart grid: Dynamic modeling and stability," in Proceedings of the 50th IEEE Conference on Decision and Control, 2011, pp. 2202-2207.

[12] H. Hindi, D. Greene, and C. Laventall, "Coordinating regulation and demand response in electric power grids: Direct and price-based tracking using multirate economic model predictive control," in Control and Optimization Methods for Electric Smart Grids, A. Chakrabortty and M. Ilić, Eds. Springer, 2012, pp. 111-131.

[13] S. Coogan, L. J. Ratliff, D. Calderone, C. Tomlin, and S. S. Sastry, "Energy management via pricing in LQ dynamic games," in Proceedings of the 2013 American Control Conference, Washington, DC, 2013, pp. $443-448$

[14] S. Maharjan, Q. Zhu, Y. Zhang, S. Gjessing, and T. Başar, "Dependable demand response management in the smart grid: A stackelberg game approach," IEEE Transactions on Smart Grid, pp. 120-132, 2013.

[15] K. Hirata, J. P. Hespanha, and K. Uchida, "Real-time pricing leading to optimal operation under distributed decision makings," in Proceedings of the 2014 American Control Conference, Portland, 2014, pp. 19521932.

[16] M. A. Pai, Energy Function Analysis for Power System Stability. Kluwer Academic Publishers, 1989.
[17] S. Boyd and L. Vandenberghe, Convex Optimization. Cambridge University Press, 2004.

[18] G. Baba et al., "Optimal operation under distributed decision makings with pricing delay," in Proceedings of the 1st Multi-symposium on Control Systems, 2014, pp. 6A3-4, in Japanese.

[19] Y. Okada, K. Hirata, and K. Uchida, "Real-time pricing leading to optimal operation and its stability analysis," in Proceedings of the 1st Multi-symposium on Control Systems, 2014, pp. 6A3-1, in Japanese.

[20] H. Saadat, Power System Analysis, 3rd ed. PSA Publishing, 2010.

[21] A. R. Bergen and V. Vittal, Power System Analysis, 2nd ed. Prentice Hall, 2000.

[22] R. D. Christie, B. F. Wollenberg, and I. Wangensteen, "Transmission management in the deregulated environment," Proceedings of the IEEE, vol. 88, no. 2, pp. 170-195, 2000.

\section{APPENDIX}

Proof: [Lemma 3] Since $\operatorname{span}\left\{\mathbb{1}_{n}\right\} \perp \operatorname{Im} M$, there exist $\theta_{\ell}$ and $\theta_{g}$ that satisfy (10b), if and only if, $r$ satisfies (15b).

Let $r$ be any constant that satisfies (15b), and let us pick $\theta_{\ell}$ and $\theta_{g}$ that satisfy (10b). We set $x=\left[\begin{array}{lll}\theta_{\ell}^{\mathrm{T}} & \theta_{g}^{\mathrm{T}} & 0\end{array}\right]^{\mathrm{T}}$. By substituting $r$ and $x$ into (14a) and (14c), we can confirm that $x$ is an equilibrium point and $\hat{z}=\left[\begin{array}{ll}0^{\mathrm{T}} & r^{\mathrm{T}}\end{array}\right]^{\mathrm{T}}$.

Proof: [Lemma 4] i) $\sum_{i=1}^{n_{g}} J_{i}\left(r^{\natural}\right) \geq \sum_{i=1}^{n_{g}} J_{i}\left(r^{*}\right)$ : Вy multiplying $\mathbb{1}_{n}^{\mathrm{T}}$ from the left to $(10 \mathrm{~b})$, since $\mathbb{1}_{n}^{\mathrm{T}} M=0$, we have that $0=\mathbb{1}_{n_{g}}^{\mathrm{T}} r^{\natural}-\mathbb{1}_{n}^{\mathrm{T}} w$. Thus, $r^{\natural}$ is a feasible solution to (15), and we have $\sum_{i=1}^{n_{g}} J_{i}\left(r^{\natural}\right) \geq \sum_{i=1}^{n_{g}} J_{i}\left(r^{*}\right)$.

ii) $\sum_{i=1}^{n_{g}} J_{i}\left(r^{\natural}\right) \leq \sum_{i=1}^{n_{g}} J_{i}\left(r^{*}\right)$ : From Lemma 3 , there exists $x^{*}$ such that

$$
\left[\begin{array}{ll}
0^{\mathrm{T}} & \left(r^{*}\right)^{\mathrm{T}}
\end{array}\right]^{\mathrm{T}}=\hat{C} x^{*}+w=\hat{C} x^{*}+w
$$

Since $\operatorname{Im} \hat{C} \subset \operatorname{Im} M$, there exits $\left[\left(\theta_{\ell}^{*}\right)^{\mathrm{T}} \quad\left(\theta_{g}^{*}\right)^{\mathrm{T}}\right]^{\mathrm{T}}$ such that $\hat{C} x^{*}=M\left[\left(\theta_{\ell}^{*}\right)^{\mathrm{T}}\left(\theta_{g}^{*}\right)^{\mathrm{T}}\right]^{\mathrm{T}}$, and, by substituting this into

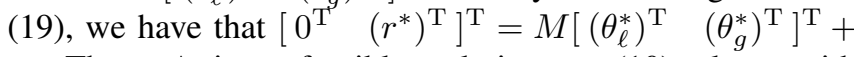
$w$. Thus $r^{*}$ is a feasible solution to (10) along with $\left[\left(\theta_{\ell}^{*}\right)^{\mathrm{T}} \quad\left(\theta_{g}^{*}\right)^{\mathrm{T}}\right]^{\mathrm{T}}$, and we have $\sum_{i=1}^{n_{g}} J_{i}\left(r^{\natural}\right) \leq \sum_{i=1}^{n_{g}} J_{i}\left(r^{*}\right)$. iii): We suppose that $r^{*} \neq r^{\natural}$. For any $0<\alpha<1$, since both of $r^{*}$ and $r^{\natural}$ are feasible solution to (15), we have $\mathbb{1}_{n_{g}}^{\mathrm{T}}\left(\alpha r^{*}+(1-\alpha) r^{\natural}\right)=\alpha \mathbb{1}_{n_{g}}^{\mathrm{T}} r^{*}+(1-\alpha) \mathbb{1}_{n_{g}}^{\mathrm{T}} r^{\natural}=$ $\alpha \mathbb{1}_{n}^{\mathrm{T}} w+(1-\alpha) \mathbb{1}_{n}^{\mathrm{T}} w=\mathbb{1}_{n}^{\mathrm{T}} w$, thus $\alpha r^{*}+(1-\alpha) r^{\natural}$ is a feasible solution to (15). Due to strict convexity of the functions $J_{i}$, we have that $\sum_{i=1}^{n_{g}} J_{i}\left(\alpha r_{i}^{*}+(1-\alpha) r_{i}^{\natural}\right)<$ $\alpha \sum_{i=i}^{n_{g}} J_{i}\left(r_{i}^{*}\right)+(1-\alpha) \sum_{i=i}^{n_{g}} J_{i}\left(r_{i}^{\natural}\right)$. From i) and ii), we have $\sum_{i=1}^{n_{g}} J_{i}\left(r^{*}\right)=\sum_{i=1}^{n_{g}} J_{i}\left(r^{\natural}\right)$ and conclude that $\sum_{i=1}^{n_{g}} J_{i}\left(\alpha r_{i}^{*}+(1-\alpha) r_{i}^{\natural}\right)<\sum_{i=1}^{n_{g}} J_{i}\left(r^{*}\right)$. This contradicts optimality of $r^{*}$, and we conclude that $r^{*}=r^{\natural}$. 